\title{
REPRESENTATION OF NUMBERS BY CASCADES
}

\author{
C. C. CHEN AND D. E. DAYKIN
}

Abstract. A cascade $C$ is defined as a sum of binomial coefficients

$$
C=\left(\begin{array}{c}
a_{h} \\
h
\end{array}\right)+\left(\begin{array}{c}
a_{h-1} \\
h-1
\end{array}\right)+\cdots+\left(\begin{array}{c}
a_{t} \\
t
\end{array}\right)
$$

where $a_{h}>a_{h-1}>\cdots>a_{t}$. In this expression, we assume that $\left(\begin{array}{l}a \\ h\end{array}\right)=0$ whenever $a<h$. Given a cascade $C$ and a sequence $\varepsilon=\left\langle\varepsilon_{h}, \varepsilon_{h-1}, \ldots, \varepsilon_{t}\right\rangle$ of signs (i.e. $\varepsilon_{i}=+1$ or -1 for each $i$ ), we define

$$
\varepsilon C=\varepsilon_{h}\left(\begin{array}{c}
a_{h} \\
h
\end{array}\right)+\cdots+\varepsilon_{t}\left(\begin{array}{c}
a_{t} \\
t
\end{array}\right) .
$$

Also, we put

$$
\alpha C=\left(\begin{array}{c}
a_{h} \\
h+1
\end{array}\right)+\left(\begin{array}{c}
a_{h-1} \\
h
\end{array}\right)+\cdots+\left(\begin{array}{c}
a_{t} \\
t+1
\end{array}\right) .
$$

In the paper, we prove that for any sequence $\left\langle n_{0}, n_{1}, \ldots, n_{s}\right\rangle$ of integers, there exist a cascade $C$ and a corresponding sequence $\varepsilon$ of signs such that $n_{i}=\varepsilon \alpha^{i} C$ for $i=0,1, \ldots, s$ where $\alpha^{0} C=C, \alpha^{1} C=\alpha C, \alpha^{2} C=\alpha\left(\alpha^{1} C\right)$, and recursively, $\alpha^{n} C=\alpha\left(\alpha^{n-1} C\right)$.

1. Introduction. Let $a_{h}, a_{h-1}, \ldots, a_{t}, h, t$ be integers with $a_{h}>a_{h-1}>\cdots$ $>a_{t}$. We shall call an expression of the form

$$
C=\left(\begin{array}{c}
a_{h} \\
h
\end{array}\right)+\left(\begin{array}{c}
a_{h-1} \\
h-1
\end{array}\right)+\cdots+\left(\begin{array}{c}
a_{t} \\
t
\end{array}\right)
$$

a cascade; $h$ is called the head, $t$ the tail, $h-t+1$ the length and $a_{h}-h$ the rank of the cascade $C$. Note that in the expression (I), each binomial coefficient

$$
\left(\begin{array}{c}
a_{i} \\
i
\end{array}\right) \text { with } a_{i}<i \text { or } i<0
$$

is equal to zero. Also the cascade is said to be normal if $a_{t} \geqslant t \geqslant 0$ but not $a_{t}=t=0$.

For each cascade $C$ as given in (I), we define new cascades $\alpha(C), \delta(C), \mu(C)$ and $\rho(C)$ respectively by:

Received by the editors May 15, 1975 and, in revised form, August 1, 1975.

AMS (MOS) subject classifications (1970). Primary 05A05, 05A10; Secondary 042A20.

(c) American Mathematical Society 1976 


$$
\begin{aligned}
& \alpha(C)=\left(\begin{array}{c}
a_{h} \\
h+1
\end{array}\right)+\left(\begin{array}{c}
a_{h-1} \\
h
\end{array}\right)+\cdots+\left(\begin{array}{c}
a_{t} \\
t+1
\end{array}\right), \\
& \delta(C)=\left(\begin{array}{c}
a_{n} \\
h-1
\end{array}\right)+\left(\begin{array}{c}
a_{h-1} \\
h-2
\end{array}\right)+\cdots+\left(\begin{array}{c}
a_{t} \\
t-1
\end{array}\right), \\
& \mu(C)=\left(\begin{array}{c}
a_{h}-1 \\
h
\end{array}\right)+\left(\begin{array}{c}
a_{h-1}-1 \\
h-1
\end{array}\right)+\cdots+\left(\begin{array}{c}
a_{t}-1 \\
t
\end{array}\right), \\
& \rho(C)=\left(\begin{array}{c}
a_{h}-1 \\
h-1
\end{array}\right)+\left(\begin{array}{c}
a_{h-1}-1 \\
h-2
\end{array}\right)+\cdots+\left(\begin{array}{c}
a_{t}-1 \\
t-1
\end{array}\right) .
\end{aligned}
$$

Recursively, for $n \geqslant 0, \alpha^{n}(C)$ means $\alpha\left(\alpha^{n-1}(C)\right)$ and $\alpha^{0}(C)=C \cdot \delta^{n}(C)$, $\mu^{n}(C)$ and $\rho^{n}(C)$ are defined likewise. It is evident that for any cascade $C$, $\delta \alpha(C)=\alpha \delta(C)=C, \mu(C)=\rho \alpha(C)$ and $\rho(C)=\delta \mu(C)$.

Again, let $C$ be the cascade given in (I) and let $\varepsilon=\left\langle\varepsilon_{h}, \varepsilon_{h-1}, \ldots, \varepsilon_{t}\right\rangle$ be a sequence of signs (i.e. each $\varepsilon_{i}$ is either +1 or -1 ). We shall denote by $\varepsilon C$ the number

$$
\varepsilon C=\varepsilon_{h}\left(\begin{array}{c}
a_{h} \\
h
\end{array}\right)+\varepsilon_{h-1}\left(\begin{array}{c}
a_{h-1} \\
h-1
\end{array}\right)+\cdots+\varepsilon_{t}\left(\begin{array}{c}
a_{t} \\
t
\end{array}\right) .
$$

Notice that whenever we write such an expression we assume the sequence and the cascade have the same length.

As far as we know, cascades were first found by Kruskal [1] and play an important role in the theory of finite sets. They have been used [1], [2], [5] to prove the Kruskal-Katona theorem, which has the Erdös-Ko-Rado theorem as a corollary. It is well known now that given $h \geqslant 1$ each positive integer has a unique representation as a cascade with head $h$ and tail $>0$. It is shown in [6] that given $c, h \geqslant 1$ each positive integer has a unique representation of the form $C-\delta^{c}(C)$, where if (I) is $C$ then $t>0$ and $a_{i} \geqslant 2 i-c$ for $h \geqslant i \geqslant t$. Results of a different kind are obtained in [6] by attaching signs to the binomial coefficients in a cascade and then forming a family of finite sets according to certain rules. In view of all these results it is natural to study the sequence $\cdots, \varepsilon \alpha^{2}(C), \varepsilon \alpha(C), C, \varepsilon \delta(C), \varepsilon \delta^{2}(C), \ldots$ and our main result is

THEOREM 1. Let $n_{1}, n_{2}, \ldots, n_{s}$ be any sequence of integers. Then for each positive integer $N$, there exist a normal cascade $C$ with rank $s-1$ and tail $\geqslant N$, and a corresponding sequence $\varepsilon$ of signs, such that

$$
n_{i}=\varepsilon \alpha^{s-i}(C) \text { for } i=1,2, \ldots, s .
$$

Conjecture. We believe that some normal $C$ and $\varepsilon$ give also $n_{i}=\varepsilon \delta^{s-i}(C)$.

2. The proof. First of all, we wish to point out that the reader may find it helpful to display the Pascal's triangle as in Figure 1. If the binomial coefficients appearing in a normal cascade are marked in the figure, then the marks resemble water flowing from right to left: 


$$
\begin{aligned}
& \cdots\left(\begin{array}{l}
2 \\
2
\end{array}\right)\left(\begin{array}{l}
1 \\
1
\end{array}\right)\left(\begin{array}{l}
0 \\
0
\end{array}\right) \\
& \text {.. }\left(\begin{array}{l}
3 \\
2
\end{array}\right)\left(\begin{array}{l}
2 \\
1
\end{array}\right)\left(\begin{array}{l}
1 \\
0
\end{array}\right) \\
& \cdots\left(\begin{array}{l}
4 \\
2
\end{array}\right)\left(\begin{array}{l}
3 \\
1
\end{array}\right)\left(\begin{array}{l}
2 \\
0
\end{array}\right)
\end{aligned}
$$

Figure 1. Pascal's Triangle

A cascade $C$ as given in (I) is called a flat cascade if $a_{h}-h=a_{h-1}$ $-(h-1)=\cdots=a_{t}-t$. It has rank $a_{h}-h$. A pattern $p_{i}$ for row $i \geqslant-1$ of length $m$ is a sequence of signs $p_{i}=\left\langle\varepsilon_{1}, \varepsilon_{2}, \ldots, \varepsilon_{m}\right\rangle$ such that for any normal flat cascade $C$ of rank $i$ and length $m$, the number $p_{i} C$ is a constant. We call this constant the value of $p_{i}$, denoted by $v\left(p_{i}\right)$. Also, we shall denote the smallest $v\left(p_{i}\right)>0$ when $p_{i}$ runs through all patterns of row $i$ by $v_{i}$ and call it the value of row $i$. It is easy to observe that $v_{0}=1, v_{1}=1$ and $v_{2}=2$ with patterns $p_{0}=\langle+1\rangle, p_{1}=\langle+1,-1\rangle$ and $p_{2}=\langle+1,-1,-1,+1\rangle$. Also, for each pattern $p_{i}=\left\langle\varepsilon_{1}, \ldots, \varepsilon_{m}\right\rangle$ for row $i$, the pattern $p_{i}-p_{i}=\left\langle\varepsilon_{1}, \ldots, \varepsilon_{m}\right.$, $\left.-\varepsilon_{1}, \ldots,-\varepsilon_{m}\right\rangle$ is a zero value pattern for row $i$. In fact, every sequence of signs is a pattern for row 0 , because every binomial coefficient is 1 .

LEMMA 1 . For $i \geqslant 0$ and each pattern $p$ for row $i$ and every proper flat cascade $C$ of rank $i$, we have $p C=p \mu(C)+p \rho(C)$.

PROOF. This follows directly from the relation

$$
\left(\begin{array}{l}
r \\
s
\end{array}\right)=\left(\begin{array}{c}
r-1 \\
s
\end{array}\right)+\left(\begin{array}{l}
r-1 \\
s-1
\end{array}\right) \text { for } r \geqslant s \geqslant 0 \text { not } r=s=0 .
$$

Lemma 2. For $i \geqslant 0$ the pattern $p$ is a zero value pattern for row $i-1$ if and only if $p$ is a pattern for row $i$.

Proof. If $p$ is a zero value pattern for row $i-1$, then for any normal flat cascade $C$ of rank $i$, we have, by Lemma 1 , that $p C=p \mu(C)+p \rho(C)$ $=p \rho(C)$. Now $\mu(C)$ is a flat cascade of rank $i-1$ which is normal except in the one trivial case $i=1$ and tail $=0$ so $p \mu(C)=0$. Therefore $p$ is a pattern for row $i$.

Conversely, if $p$ is a pattern for row $i$, then for any flat cascade $C$ of rank $i-1$ and tail $\geqslant 1$, we have $p D=p \mu(D)+p \rho(D)$ where $D$ is the flat cascade of rank $i$ with $\mu(D)=C$. But then, $p C=p \mu(D)=0$ since $p(D)=p \rho(D)$. Repetition shows $p B=0$ for any flat cascade $B$ of rank $i-2$ and tail $\geqslant 2$. Eventually we find that $p$ is a zero value pattern for row 0 . Then by the first part of the proof we know that $p$ is a pattern for row 1 and so must be a zero value pattern. Continuing we find that $p$ is a zero value pattern for row $i-1$ as required.

COROLlaRy. There exists a pattern for row $i$ for each $i=0,1,2, \ldots$. 
LEMMA 3. If $p_{1}, p_{2}$ are patterns for row $i$ with values $u_{1}$ and $u_{2}$ respectively, then there exists a pattern $p$ for row $i$ with value $g=$ g.c.d. $\left(u_{1}, u_{2}\right)$.

Proof. We can write $g=\lambda_{1} u_{1}+\lambda_{2} u_{2}$ for suitable integers $\lambda_{1}, \lambda_{2}$. Then all we need to do is take a pattern $q$ consisting of $\left|\lambda_{1}\right|$ copies of $p_{1}$ and $\left|\mu_{2}\right|$ copies of $p_{2}$ with appropriate signs.

As pointed out before, we have $v_{0}=1, v_{1}=1, v_{2}=2$, arising from the patterns $p_{0}=\langle+1\rangle, p_{1}=\langle+1,-1\rangle$ and $p_{2}=\langle+1,-1,-1,+1\rangle$ respectively. Let us try to estimate the value $v_{3}$. Let $C$ be a flat cascade of length 4 , rank 3 and tail sufficiently large. Then we have $p_{2} C-p_{2} \rho(C)=p_{2} \mu(C)$ by Lemma 1. But since $\mu(C)$ is a cascade of rank 2 , we have $p_{2} \mu(C)=v_{2}$, which implies that

$$
p_{2} C-p_{2} \rho(C)=v_{2}
$$

Similarly,

$$
p_{2} \rho(C)-p_{2} \rho^{2}(C)=v_{2}, \quad p_{2} \rho^{2}(C)-p_{2} \rho^{3}(C)=v_{2}
$$

and

$$
p_{2} \rho^{3}(C)-p_{2} \rho^{4}(C)=v_{2}
$$

Therefore

$$
p_{2} C-p_{2} \rho^{4}(C)=4 v_{2}
$$

i.e.

$$
\left(p_{2}-p_{2}\right)\left(C+\rho^{4}(C)\right)=4 v_{2}=8,
$$

which implies that $p_{2}-p_{2}$ is a pattern for row 3 with value 8 . This argument can easily be generalized to obtain

LEMMA 4. There exists a pattern for row $i$ with value $2^{\left(\begin{array}{l}i \\ 2\end{array}\right)}$ for $i=0,1,2, \ldots$

COROllary. The value $v_{i}$ of row $i$ divides $2^{\left(\begin{array}{c}i \\ 2\end{array}\right)}$.

Proof. This follows immediately from Lemmas 3 and 4.

We shall now prove Theorem 1 by induction on $s$. For $s=1$, we need only choose a flat cascade of rank 0 say

$$
C=\left(\begin{array}{l}
a \\
a
\end{array}\right)+\left(\begin{array}{l}
a-1 \\
a-1
\end{array}\right)+\cdots+\left(\begin{array}{l}
a-\left|n_{s}\right|+1 \\
a-\left|n_{s}\right|+1
\end{array}\right) \text { with } a-\left|n_{s}\right|+1 \geqslant N
$$

The corresponding sequence of signs is either all positive or all negative depending on whether $n_{s}$ is positive or not. Assume that the theorem holds for any sequence of $s-1$ integers and consider the sequence $\left\langle n_{1}, n_{2}, \ldots, n_{s}\right\rangle$. Applying the induction hypothesis to $n_{1}, \ldots, n_{s-1}$, there is a proper cascade 


$$
D=\left(\begin{array}{c}
a_{h} \\
h
\end{array}\right)+\cdots+\left(\begin{array}{c}
a_{t} \\
t
\end{array}\right)
$$

with rank $s-2$ and tail $\geqslant N+v_{s-1}$, and also a sequence $\varepsilon^{*}=\left\langle\varepsilon_{h}, \ldots, \varepsilon_{t}\right\rangle$ of signs such that $\varepsilon^{*} D=n_{s-1}, \varepsilon^{*} \alpha(D)=n_{s-2}, \ldots, \varepsilon^{*} \alpha^{s-2}(D)=n_{1}$. Let $C_{1}$ $=\delta D$ and $m=n_{s}-\varepsilon^{*} C_{1}$. Without loss of generality, we may assume that $m \geqslant 0$. Let $[m / v]=k$ where $v=v_{s-1}$ and let $p=\left\langle p_{1}, \ldots, p_{l}\right\rangle$ be a pattern for row $s-1$ giving the value $v$. Now, let $C_{2}$ be the flat cascade of rank $s-1$ with tail $h$ and length $k l$ and let $C_{3}$ be the flat cascade of rank 0 with head $(t-2)$ and length $m-k v$. Finally in the obvious way put

$$
\varepsilon=+p \cdots+p+\varepsilon^{*}+1 \cdots+1
$$

and $C=C_{2}+C_{1}+C_{3}$. Then $C$ is evidently a cascade of rank $s-1$ with $\varepsilon C=n_{s}$. Also by Lemma $2, p \alpha^{j} C_{2}=0$ for $j=1,2, \ldots$ Thus $\varepsilon \alpha(C)=n_{s-1}$, $\varepsilon \alpha^{2}(C)=n_{s-2}, \ldots, \varepsilon \alpha^{s-1}(C)=n_{1}$ completing the proof.

3. Remarks. (a) The proof of Theorem 1 in fact provides us with an algorithm to construct $C$ and the corresponding sequence of signs. It is sufficient for the flat cascade of rank 0 with which we start to have a tail greater than

$$
2^{\left(\frac{1}{2}\right)}+2^{\left(\frac{2}{2}\right)}+\cdots+2^{(s-1)} \text {. }
$$

(b) The cascade of Theorem 1 can be chosen in infinitely many different ways.

(c) We omit the proof of

THEOREM 2. Let $n_{1}, n_{2}, \ldots, n_{s}$ be any sequence of integers. Then for each positive integer $N$ there exists a flat cascade $C$ with rank 0 and tail $\geqslant N$, and $a$ corresponding sequence $\varepsilon$ of signs such that

$$
\left|n_{i}-\varepsilon \delta^{s-i}(C)\right| \leqslant 2^{\left(\frac{i}{2}\right)-1} \text { for } i=1,2, \ldots, s .
$$

\section{REFERENCES}

1. J. B. Kruskal, The number of simplices in a complex, Mathematical Optimization Techniques, Univ. of California Press, Berkeley, 1963, pp. 251-278. MR 27 \#4771.

2. G. Katona, A theorem of finite sets, Theory of Graphs (Proc. Colloq., Tihany, 1966), Academic Press, New York, 1968, pp. 187-207. MR 45 \#76.

3. D. E. Daykin, Jean Godfrey and A. J. W. Hilton, Existence theorems for Sperner families, J. Combinatorial Theory Ser. A 17 (1974), 245-251.

4. D. E. Daykin, A simple proof of the Kruskal-Katona theorem, J. Combinatorial Theory Ser. A 17 (1974), 252-253.

5. - Cascade algorithms giving Katona-type inequalities, Nanta. Math. (to appear).

6. - The average size set in an antichain, Nanta. Math. (to appear).

Department of Mathematics, Nanyang University, Singapore

Department of Mathematics, University of Reading, Reading, England 